\title{
Semaphorin 3E Alleviates Hallmarks of House Dust Mite-Induced Allergic Airway Disease
}

\author{
Hesam Movassagh, ${ }^{*}$ Lianyu Shan, ${ }^{*}$ Jonathan S. Duke-Cohan ${ }^{\dagger \S}$ Andrew J. Halayko, ${ }^{\ddagger}$ Jude E. Uzonna, ${ }^{*}$ and Abdelilah S. Gounni ${ }^{\star}$
}

From the Departments of Immunology* and Physiology and Pathophysiology, ${ }^{\ddagger}$ Rady Faculty of Health Sciences, Max Rady College of Medicine, University of Manitoba, Winnipeg, Manitoba, Canada; and the Department of Medical Oncology, ${ }^{\dagger}$ Laboratory of Immunobiology, Dana-Farber Cancer Institute, Boston, Massachusetts; and the Department of Medicine, ${ }^{\S}$ Harvard Medical School, Boston, Massachusetts

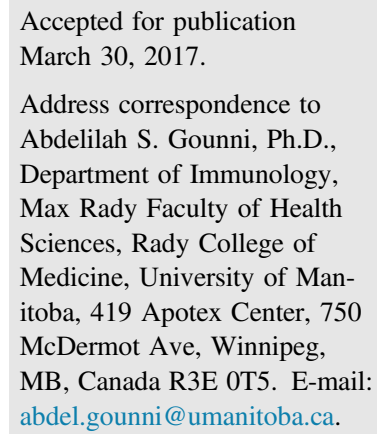

\begin{abstract}
Semaphorins are an essential family of guidance cues ubiquitously expressed in various organs, which play diverse developmental, homeostatic, and pathological roles. Semaphorin 3E (Sema3E), initially identified as a neuronal chemorepellent, is involved in the regulation of cell migration, proliferation, and angiogenesis. However, expression and function of Sema3E in allergic asthma has not been extensively investigated. We determined the expression of Sema3E in the airways and its effect on airway inflammation, hyperresponsiveness, and remodeling as pathological features of allergic asthma provoked by house dust mite in vivo. Our data indicate that exposure to house dust mite markedly reduces Sema3E expression in mouse airways. More important, replenishment of Sema3E by intranasal administration of exogenous Sema3E protects mice from allergic asthma by reducing eosinophilic inflammation, serum IgE level, and T helper cell 2/T helper cell 17 cytokine response. The regulatory effect of Sema3E on cytokine response was sustained on allergen recall response in the lymph nodes and spleen. Furthermore, goblet cell hyperplasia, collagen deposition, and airway hyperresponsiveness were significantly diminished on Sema3E treatment. The inhibitory effect of Sema3E was associated with a reduction of pulmonary $\mathrm{CD}_{11} \mathrm{~b}^{+}$conventional dendritic cells and regulation of $\mathrm{CD}^{+}{ }^{+} \mathrm{T}$-cell cytokine response. Collectively, our data represent a novel approach to treating allergic asthma via regulation of immune response to house dust mite. (Am J Pathol 2017, 187: 1566-1576; http://dx.doi.org/10.1016/ j.ajpath.2017.03.008)
\end{abstract}

Asthma is a multifaceted disease of the airways associated with chronic inflammation, bronchoconstriction, remodeling, and airway hyperresponsiveness (AHR). ${ }^{1}$ The prevalence of allergic asthma has been remarkably increasing for the past decades. ${ }^{2}$ Therefore, development of new strategies to treat allergic asthma, in particular severe refractory form of the disease, is an urgent unmet clinical need. However, novel therapeutic approaches fail to significantly alleviate the cardinal features of asthma, especially AHR and remodeling. $^{3-5}$

Continuous exposure with innocuous aeroallergens, such as house dust mite (HDM), deviates the immune system toward a granulocytic rich response. It perpetuates the airway inflammation that leads to a massive structural alteration of the airways and subsequently AHR as the hallmark of disease pathology. ${ }^{6}$ However, the mechanisms underlying airway remodeling and AHR could spontaneously develop independent of inflammatory response. ${ }^{7,8}$ Also, a considerable portion of asthmatic patients have a $\mathrm{T}$ helper cell (Th) 2-low noneosinophilic phenotype that poorly responds to current anti-inflammatory treatments (eg, corticosteroids). 5,9,10 Thus, efficient therapeutic strategies for allergic asthma should be able to target various arms of the disease, including both inflammatory and structural compartments of the airways.

Semaphorins are guidance cues that play diverse roles, such as immune regulation, angiogenesis, cell

\footnotetext{
Supported by Canadian Institutes of Health Research grant MOP 115115, National Sciences and Engineering Research Council grant RG PIN/ 386289, and a Children Hospital Research Foundation grant (A.S.G.). H.M. was supported by Research Manitoba-Children's Hospital Research Institute of Manitoba Studentship.
}

Disclosures: None declared. 
proliferation, and migration. ${ }^{11-13}$ Various semaphorins have been reported to exert proinflammatory or antiinflammatory responses in a context-dependent manner. ${ }^{14-17}$ For instance, Semaphorin 3E (Sema3E) is able to dualistically promote or suppress inflammation in the obesity model ${ }^{18}$ or atherosclerotic plaques, ${ }^{19}$ respectively, via regulation of macrophage functions. Sema3E is ubiquitously expressed in different cell types, including adipocytes, ${ }^{18}$ macrophages, ${ }^{19}$ tumor cells, ${ }^{20}$ hepatocytes, ${ }^{21}$ osteoblasts, ${ }^{22}$ and dendritic cells. ${ }^{23}$ It is involved in essential cellular functions that are dysregulated in allergic asthma. ${ }^{24-27} \mathrm{We}$ have previously reported that in vitro treatment of human airway smooth muscle (ASM) cells with Sema3E inhibits growth factor-induced proliferation and migration considered as fundamental mechanisms contributing to airway remodeling. ${ }^{28}$ This effect was associated with suppression of mitogenactivated protein kinase, phosphatidylinositol 3-kinase, and Ras-related C3 botulinum toxin substrate 1 GTPase signaling. ${ }^{28}$ Furthermore, we have recently revealed that genetic abrogation of Sema3E in mice leads to a hypergranulocytic phenotype, which is associated with enhanced AHR, remodeling, and Th2/Th17 inflammation. ${ }^{29}$ Sema3E was also shown to reduce neutrophil recruitment to the airways on HDM reexposure. ${ }^{30}$ However, the therapeutic potential of Sema3E in allergic asthma has remained to be understood.

Our goal was first to determine the expression of Sema3E in the airways and then to address the in vivo effect of Sema3E on allergic airway inflammation, remodeling, and AHR using the HDM model of the disease. We also aimed to understand the mechanisms underlying Sema3E effects on the allergic asthma model. We found that Sema3E expression was down-regulated in murine airways on HDM sensitization and challenge. Administration of exogenous recombinant Sema3E protected mice from HDM-induced airway inflammation, mucus overproduction, collagen deposition, and AHR. The mechanism underlying the Sema3E effect on the HDM mouse model of allergic asthma was mediated, at least in part, via regulating cytokine response, pulmonary dendritic cell (DC) recruitment, and function. Taken together, our findings reveal a major regulatory role of Sema3E in allergic asthma.

\section{Materials and Methods}

\section{Animals}

Female 6- to 8-week-old BALB/c mice were purchased from the Central Animal Care Services at the University of Manitoba (Winnipeg, MB, Canada). All of the mice were maintained at the Central Animal Care Services facility under specific pathogen-free conditions and used according to the guidelines stipulated by the Canadian Council for Animal Care.

\section{House Dust Mite Exposure Model}

Lyophilized HDM protein extract was obtained from Greer Laboratories (Lenoir, NC), which was reconstituted in sterile saline as $2.5 \mathrm{mg} / \mathrm{mL}$ stock concentration before treatment. A working concentration $(25 \mu \mathrm{g}$ per mouse in 35 $\mu \mathrm{L}$ of saline) was freshly prepared, and an acute model of the disease was established via intranasal administration under gaseous anesthesia for 5 days per week during 2 consecutive weeks. ${ }^{31}$ Recombinant mouse Sema3E-Fc (10 $\mu \mathrm{g} / \mathrm{kg}$ in sterile phosphate-buffered saline) was administered through the intranasal route 1 hour before each HDM exposure. The control group received sterile saline- $\mathrm{Fc}$ at the same time points. All experiments were performed 48 hours after the last HDM exposure, unless otherwise indicated. Murine Sema3E-Fc recombinant protein was produced as fusion protein $\mathrm{N}$-terminal to a functional mouse $\gamma 2 \mathrm{c} \mathrm{Fc}$ domain. Complete cDNA was amplified from total RNA of a mouse brain, ligated into pFUSE-mfc1 vector, and electroporated into CHO cells. Finally, secreted Sema3E-Fc protein was purified from the conditioned media by protein A-affinity chromatography, as described previously. ${ }^{24}$

\section{Immunofluorescence}

Formalin-fixed lung tissues were paraffin embedded, and sections (5 $\mu \mathrm{m}$ thick) were prepared, dewaxed in xylene, and rehydrated through graded concentrations of alcohol to water, and then boiled for 10 minutes in sodium citrate buffer. Then, sections were incubated with blocking buffer for 1 hour at room temperature. Rabbit anti-Sema3E antibody $(2.5 \mu \mathrm{g} / \mathrm{mL}$; Abcam, Cambridge, MA) or isotype control IgG (Jackson ImmunoResearch Laboratories, West Grove, PA) was added, and sections were incubated overnight at $4^{\circ} \mathrm{C}$. Slides were then washed twice with tris-buffered saline, followed by incubation with Alexa488-conjugated anti-rabbit secondary antibody for 1 hour at room temperature away from light. After an extensive wash with tris-buffered saline, slides were finally mounted with a DAPI-containing antifade reagent (Life Technologies Inc., Burlington, ON, Canada) and visualized by Axioskop 2 mot plus microscope using AxioVision software version 4.8 (Carl Zeiss, Inc., Thornwood, NY). Integrated density of Sema3E immunostaining in the airways was compared between saline and HDM-challenged mice by ImageJ version $1.51 \mathrm{j} 8$ (NIH, Bethesda, MD; http://imagej.nih.gov/ij).

\section{Airway Responsiveness}

Mice were anesthetized and cannulated via the trachea 48 hours after the last HDM challenge. An increasing gradient of methacholine dose was administered intratracheally, and AHR parameters, including airway resistance, tissue resistance, and tissue elastance, were measured by using a FlexiVent small animal ventilator system (SCIREQ, Montreal, QC, Canada). 
A
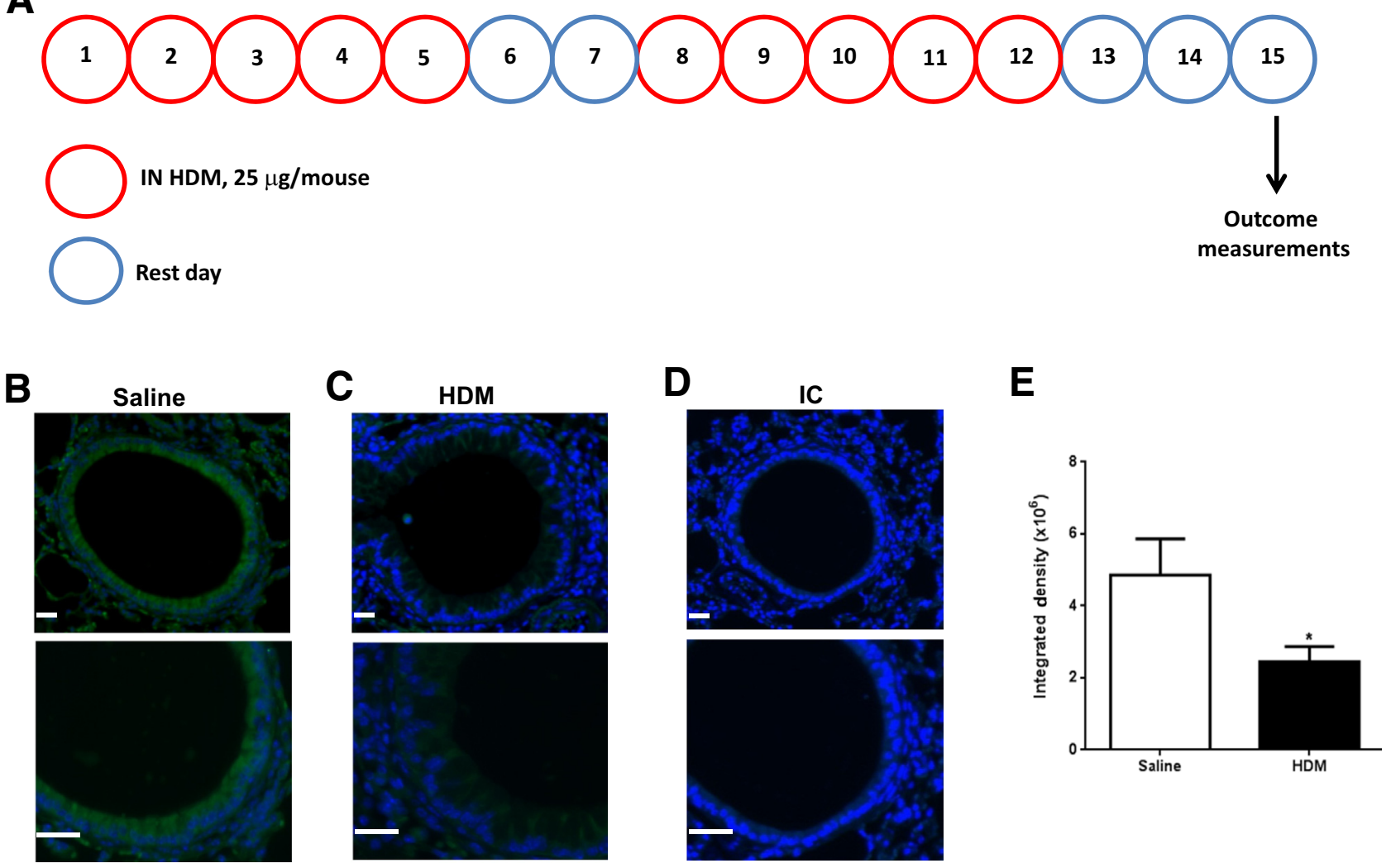

\section{Sema3E \\ DAPI}

Figure 1 Sema3E expression is reduced in the airways of HDM-exposed mice. A: Allergic airway disease model was established by intranasal (IN) administration of HDM for 2 weeks. B and C: Mouse Sema3E expression was investigated by performing immunofluorescence staining on airway sections obtained from mice exposed to either saline (B) or HDM (C). D: Staining with isotype control (IC) antibody does not show cross-reactivity. Sema3E expression was visualized with $\times 100$ and $\times 200$ magnifications after incubation of tissue sections with anti-Sema3E-specific primary antibody, fluorescein isothiocyanate-labeled secondary antibody, and counterstaining of nuclei with DAPI. E: Intensity of Sema3E staining was quantified by measuring fluorescence-integrated density. Data are expressed as means \pm SEM. $n=4$ mice per group (B-E). ${ }^{*} P<0.05$ versus saline. Scale bars: $20 \mu \mathrm{m}$ (B-D, top row); $50 \mu \mathrm{m}$ (B-D, bottom row). Original magnifications: $\times 100$ (B-D, top row); $\times 200$ (B-D, bottom row).

\section{Preparation and Processing of BAL Fluid}

Bronchoalveolar lavage (BAL) was performed with two instillations of $1 \mathrm{~mL}$ of sterile saline containing $0.1 \mathrm{mmol} / \mathrm{L}$ EDTA. Red blood cells were lysed using ammoniumchloride-potassium buffer. Total cells in BAL fluid (BALF) were spun down at $300 \times g / 4^{\circ} \mathrm{C}$ for 5 minutes, and supernatants were stored at $-80^{\circ} \mathrm{C}$ to measure cytokines. Cytospins from BALF cells were prepared and stained, and different inflammatory cell types were characterized morphologically and counted by two individuals in a blind manner (H.M. and L.S.).

\section{Lung Histology}

Dissected left lobes of mouse lungs were inflated, fixed, and embedded in paraffin. Then, sections were stained with hematoxylin and eosin, periodic acid-Schiff, and Sirius red for assessing the presence of airway inflammation, mucus overproduction, and collagen deposition, respectively. Slides were quantified by pathological scoring from 0 (the lowest staining intensity) to 5 (the highest staining intensity) in a blind manner (H.M. and L.S.).

\section{Quantification of Cytokines and Immunoglobulins}

An enzyme-linked immunosorbent assay was performed to measure IL-4, IL-5, IL-9, IL-12, IL-17A, and interferon (IFN)- $\gamma$ in BALF supernatants, according to the manufacturer's instructions. Plates were read with SpectraMax plate reader and analyzed with SoftMax Pro software version 5.4.1 (Molecular Devices, Sunnyvale, CA). All cytokine enzyme-linked immunosorbent assay kits were from BioLegend (San Diego, CA). A similar procedure was performed on supernatants obtained from the culture of mediastinal lymph node (MLN) cells, splenocytes, or coculture of lung DC subsets and splenic T cells 72 hours after HDM or vehicle stimulation. 
A

HDM: $25 \mu \mathrm{g} / \mathrm{mouse}$, IN

Sema3E-Fc: $10 \mu \mathrm{g} / \mathrm{kg}$, IN, 1 hour before HDM

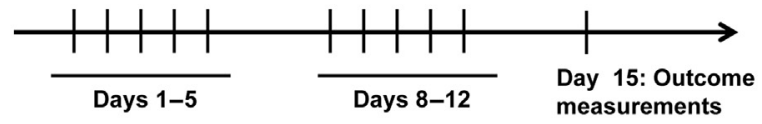

C

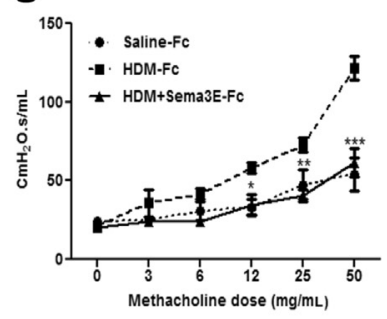

D

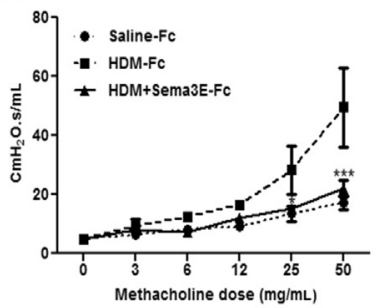

B

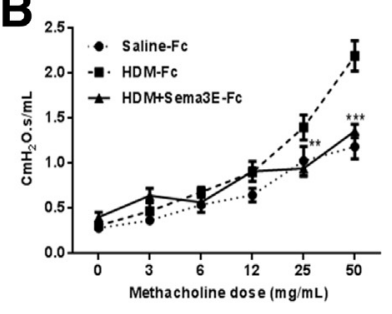

E

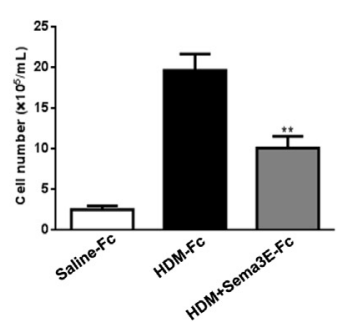

$\mathbf{F}$

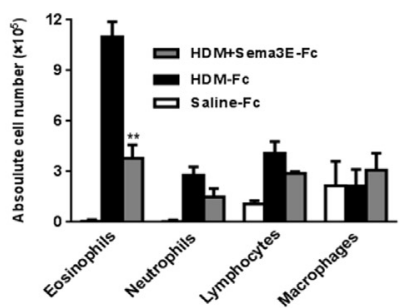

Figure 2 Sema3E prevents HDM-induced airway hyperresponsiveness (AHR) and recruitment of inflammatory cells to the airways. A: Exogenous recombinant Sema3E-Fc was administered intranasally (IN) 1 hour before each HDM exposure for 2 weeks. B-F: Sema3E treatment hampers HDM-induced airway resistance (B), tissue resistance (C), and tissue elastance (D). Total inflammatory cells (E) and eosinophils (F) are reduced in BALF on Sema3E treatment compared to those of HDM-challenged mice. Data are expressed as means \pm SEM. $n=3$ to 5 mice per group $(\mathbf{B}-\mathbf{F}) .{ }^{*} P<0.05,{ }^{* *} P<0.01$, and ${ }^{* * *} P<0.001$ versus HDM-Fc.

Serum was obtained from the mice exposed intranasally with saline-Fc or HDM-Fc with or without Sema3E-Fc. Total and HDM-specific IgE and IgG1 levels were quantified using commercial kits, according to the manufacturer's instructions (Southern Biotech, Birmingham, AL), as we described previously. ${ }^{32}$

\section{Flow Cytometric Analysis of Intracellular Cytokines}

Briefly, MLN or spleen was collected and a single-cell suspension was prepared by using a cell strainer. The cells were resuspended at a concentration of $4 \times 10^{6}$ cells $/ \mathrm{mL}$ in Dulbecco's modified Eagle's medium supplemented with $10 \%$ fetal bovine serum, $2 \mathrm{mmol} / \mathrm{L}$ L-glutamine, $100 \mathrm{U} / \mathrm{mL}$ penicillin, $100 \mu \mathrm{g} / \mathrm{mL}$ streptomycin, and $5 \times 10^{-5} \mathrm{mmol}$ 2-mercaptoethanol, plated in 24-well tissue culture plates. Then, MLN or spleen cells were incubated with a freshly prepared cocktail containing $50 \mathrm{ng} / \mathrm{mL}$ phorbol myristate acetate, $500 \mathrm{ng} / \mathrm{mL}$ ionomycin, and $10 \mu \mathrm{g} / \mathrm{mL}$ brefeldin $\mathrm{A}$, all from Sigma-Aldrich (Oakville, ON, Canada) for 4 hours at $37^{\circ} \mathrm{C}$ and $5 \% \mathrm{CO}_{2}$. Extracellular staining was performed by using anti-mouse CD3 e-Fluor 450 (clone 17A2) and CD4-fluorescein isothiocyanate (clone RM4-5), both from eBioscience (San Diego, CA). Fixed and surface-stained MLN cells were permeabilized with $0.1 \%$ saponin in flow cytometry buffer and then stained with specific fluorochrome-conjugated monoclonal antibodies, including anti-mouse IFN- $\gamma$-phosphatidylethanolamine (clone XMG1.2) and IL-4-allophycocyanin (clone 11B11) from eBioscience. Samples were acquired on a FACSCanto II (BD Biosciences, San Jose, CA) and analyzed using FlowJo software version 10 (Tree Star Inc., Ashland, OR).

\section{Analysis of Lung DC Subsets}

Major pulmonary DC subsets from the lungs were characterized 3 days after intranasal exposure with a single high dose of HDM (100 $\mu \mathrm{g} /$ mouse). ${ }^{33}$ Sema3E-Fc or saline-Fc was administered 1 hour before HDM sensitization. Briefly, lungs were minced and enzymatically digested by 1 $\mathrm{mg} / \mathrm{mL}$ collagenase IV and $0.5 \mathrm{mg} / \mathrm{mL}$ deoxyribonuclease I (Worthington Biochemical, Lakewood, NJ), followed by red blood cell lysis and Fc blocking. Then, DCs were stained by anti-mouse F4/80-fluorescein isothiocyanate (clone BM8; eBioscience), anti-mouse CD11c-allophycocyanin (clone 418; eBioscience), MHCII eFluor 450 (clone M5/ 114.15.2; eBioscience), CD11b- phosphatidylethanolamineCy7 (clone M1/70; BioLegend), and CD103-PerCP-Cy5.5 (clone 2E7; BioLegend) antibodies. Finally, pulmonary $\mathrm{CD}_{11 b^{+}}$or $\mathrm{CD}_{103}{ }^{+} \mathrm{DC}$ subsets (pregated as nonautofluorescent $\mathrm{F} 4 / 80^{-} \mathrm{CD} 11 \mathrm{c}^{+} \mathrm{MHCII}^{\mathrm{hi}}$ ) were sorted using a BD FACSAria-III Digital Cell Sorter (BD Biosciences) and co-cultured with splenic $\mathrm{T}$ cells in 1:10 ratios for 72 hours to measure the cytokine response.

\section{Statistical Analysis}

GraphPad Prism 5.0 software (Graphpad Software Inc., La Jolla, CA) was used for statistical analysis, and values were presented as the means \pm SEM of at least three independent experiments. Depending on the number of groups and treatments, data were analyzed by unpaired $t$-test, one- or two-way analysis of variance, followed by the Bonferroni's multiple comparisons post hoc test. Differences were considered to be statistically significant at $P \leq 0.05$. 

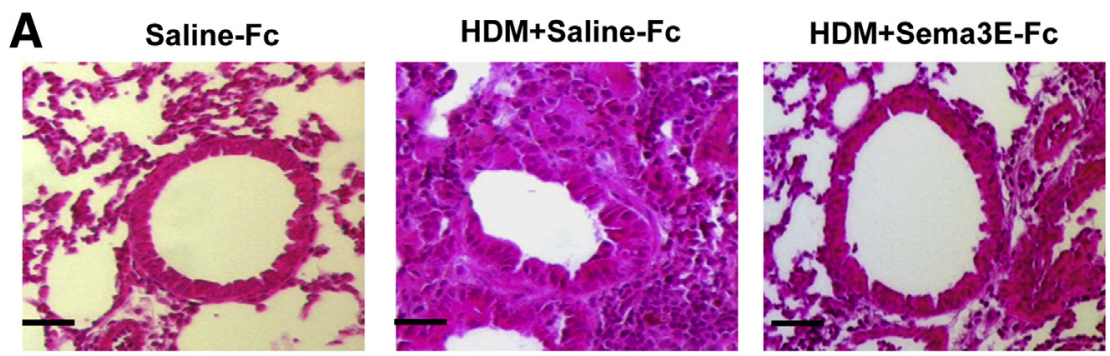

C
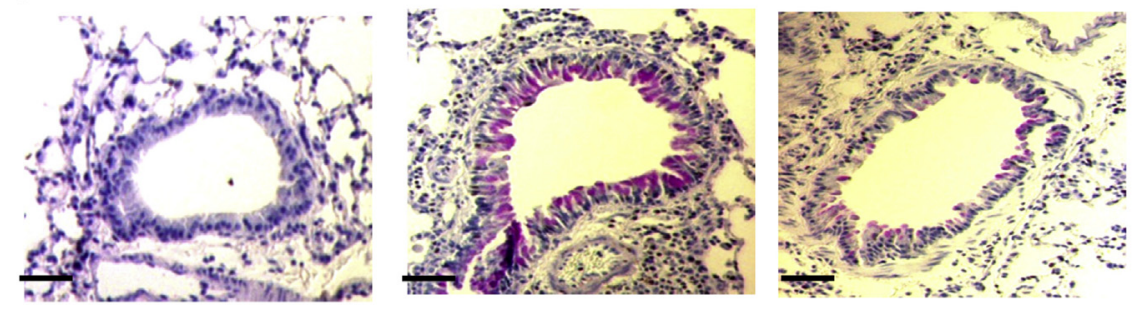

$\mathbf{E}$
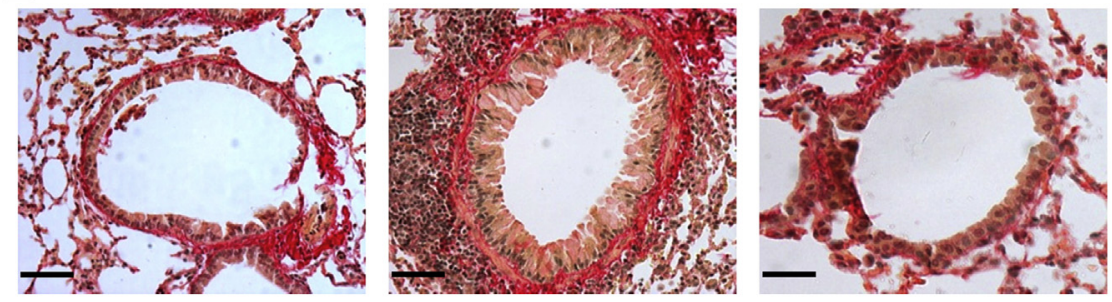

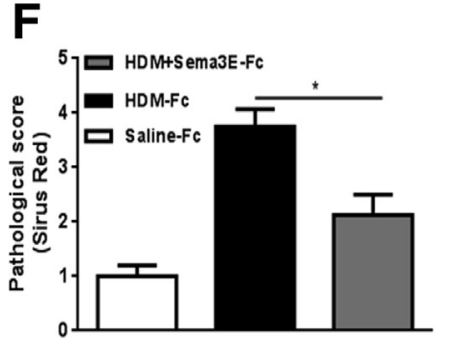

Figure 3 Sema3E reduces HDM-induced pulmonary inflammation and remodeling. Lung inflammation (A and B), mucus overproduction (C and D), and collagen deposition (E and $\mathbf{F}$ ) were histologically evaluated in lung tissue sections by performing hematoxylin and eosin (H\&E), periodic acid-Schiff (PAS), and Sirius red staining, respectively. Statistical comparison was performed between HDM plus saline-Fc and HDM plus Sema3E-Fc groups. All data represent two independent experiments. Data are expressed as means \pm SEM. $n=3$ to 5 mice per group $(\mathbf{A}-\mathbf{F}) .{ }^{*} P<0.05$, ${ }^{* \star} P<0.01$. Scale bars $=50 \mu \mathrm{m}(\mathbf{A}, \mathbf{C}$, and $\mathbf{E})$.

\section{Results}

HDM Exposure Down-Regulates Sema3E Expression in Airways

Because repeated exposure to HDM induces AHR, inflammation, and tissue remodeling, we exposed Balbc mice with this clinically relevant allergen for 2 consecutive weeks (Figure 1A). ${ }^{31,34,35}$ Then, Sema3E immunoreactivity was assessed in the allergic airways and compared with that of the saline-treated control group. Immunofluorescence staining on lung tissue sections revealed that Sema3E is highly expressed in saline-treated mice, especially in the airway epithelium (Figure 1B). In contrast, HDM sensitization and challenge dramatically decreased Sema3E expression (Figure 1C). Staining with isotype control antibody confirmed the specificity of Sema3E immunoreactivity (Figure 1D). Intensity of Sema3E immunostaining in the airways was significantly reduced on HDM exposure (Figure 1E). Decreased expression of Sema3E on HDM challenge suggests an unknown key role of this protein in airway homeostasis, which is impaired in asthmatic conditions.
Sema3E Treatment Prevents HDM-Induced AHR, Remodeling, and Inflammation

We previously reported an inhibitory effect of Sema3E on ASM cell proliferation and migration in vitro ${ }^{28}$ and also a protective role of this protein in the HDM model of asthma using Sema $3 e^{-1-}$ mice. ${ }^{29,30}$ These findings combined with decreased expression of Sema3E (Figure 1) encouraged us to examine whether Sema3E has a potential therapeutic effect on allergic asthma in vivo. Therefore, we first treated mice with either Sema3E-Fc or saline-Fc, followed by intranasal HDM or saline exposure 1 hour later for 2 consecutive weeks (Figure 2A). We observed a significant decrease in HDM-induced airway resistance (Figure 2B) as a key measure of lung function in mice that received Sema3E-Fc before HDM exposure compared to those that received saline-Fc alone. Similarly, both tissue resistance (Figure 2C) and tissue elastance (Figure 2D) were decreased on Sema3E treatment. In addition, mice treated with Sema3E-Fc exhibited a reduced number of total inflammatory cells (Figure 2E), particularly eosinophils and, to some extent, neutrophils (Figure 2F) in BALF compared to 

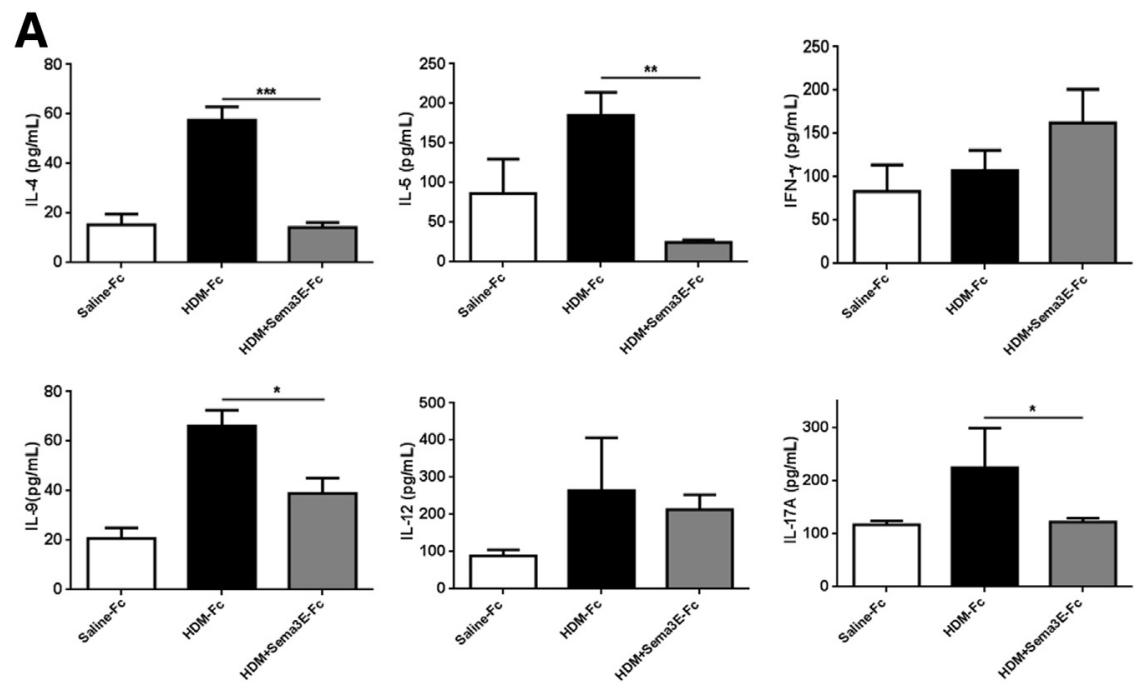

Figure 4 Sema3E treatment regulates allergic cytokine response. BALF samples were collected from HDM-exposed mice with or without Sema3E treatment and also naive mice, then subjected to enzyme-linked immunosorbent assay to measure different cytokines. A: HDM-mediated secretion of IL-4, IL-5, IL-9, and IL-17A into the airways decreases on Sema3E treatment, but interferon (IFN) $-\gamma$ level increases. B-E: Analysis of mediastinal lymph node (MLN) single-cell suspension by flow cytometry reveals a significant inhibitory effect of Sema3E on HDM-induced IL-4 (B and C) but not IFN- $\gamma$ production (D and $\mathbf{E}$ ). Data are expressed as means \pm SEM. All data represent two independent experiments. $n=3$ to $5(\mathbf{A}-\mathbf{E})$. ${ }^{*} P<0.05,{ }^{* *} P<0.01$, and ${ }^{* *}{ }^{*} P<0.001$ versus HDM.
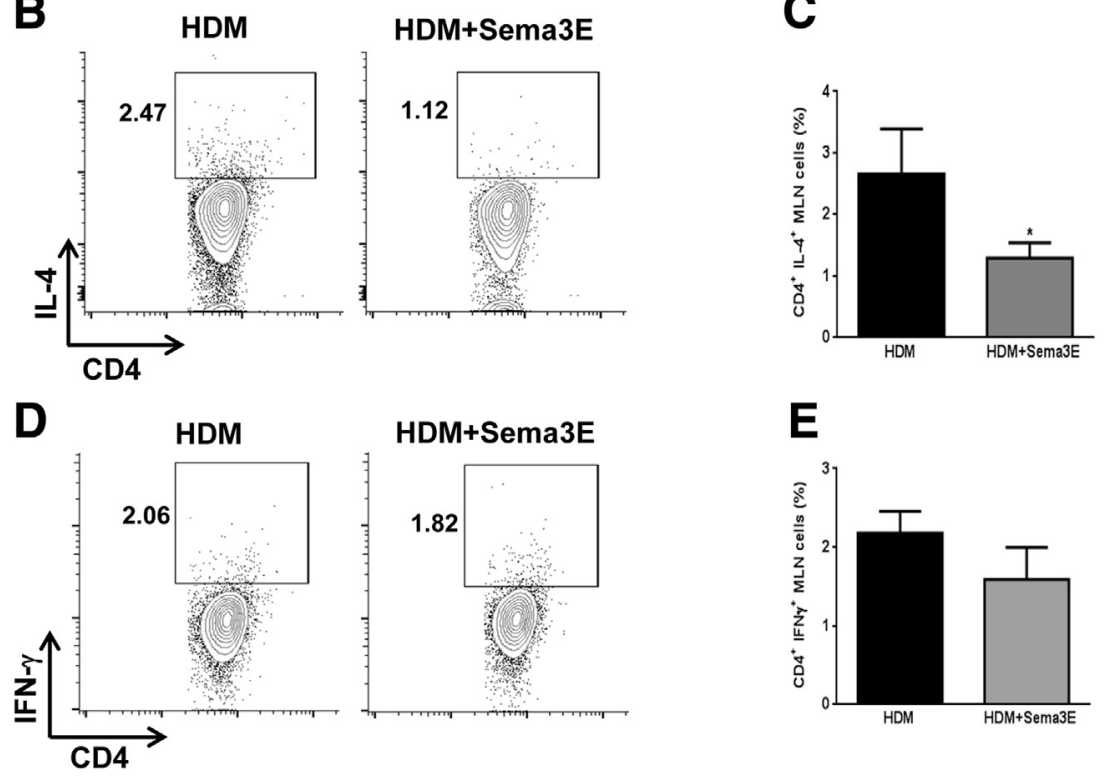

HDM+Sema3E

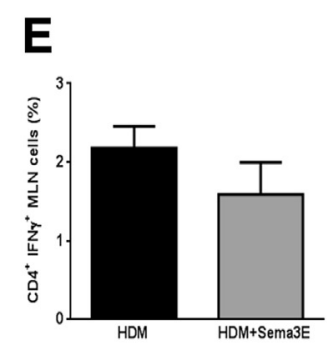

HDM-Fc-treated controls. The inhibitory effect of Sema3E on HDM-induced lung leukocytic infiltration was confirmed by hematoxylin and eosin staining (Figure 3, A and B). Mucus secretion and collagen deposition, two critical facets of airway remodeling in allergic asthma, were significantly decreased by Sema3E administration, as evaluated by periodic acid-Schiff (Figure 3, C and D) and Sirius red staining (Figure 3, E and F) on lung tissue sections, respectively. Together, these data suggest that Sema3E treatment modulates airway inflammation, remodeling, and AHR provoked by HDM.

\section{Sema3E Reduces Cytokine and Antibody Responses during an Allergic Airway Disease}

We then investigated the effect of Sema3E-Fc treatment on cytokine response. Compared to the mice given only saline$\mathrm{Fc}$ before HDM challenge, Sema3E-Fc-treated mice showed a significant decrease of IL-4, IL-5, IL-9, and IL-17A. However, IL-12 level in the BALF was not significantly altered on Sema3E-Fc treatment, whereas Sema3E-Fc treatment increased IFN- $\gamma$ level in the BALF (Figure 4A).

The effect of Sema3E-Fc treatment on Th1/Th2 cytokine response was further investigated by performing flow cytometry on lung-draining MLN ex vivo. Sema3E-Fc decreased HDM-induced intracellular production of IL-4 in $\mathrm{CD}^{+}$MLN cells (Figure 4, B and C). However, unlike BALF data, Sema3E-Fc did not change intracellular IFN- $\gamma$ production in $\mathrm{CD}_{4}^{+}$MLN cells (Figure 4, D and E). In vivo administration of Sema3E-Fc decreased IL-4 (Supplemental Figure S1, A and B) and increased IFN- $\gamma$ (Supplemental Figure S1, C and D) intracellular production in $\mathrm{CD}^{+}$ spleen cells. Collectively, our data suggest that Sema3E's role in allergic asthma is mediated, at least partially, by the orchestration of inflammatory cytokine response. 
A

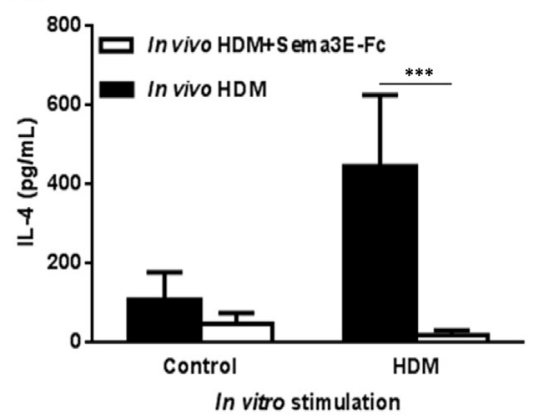

C

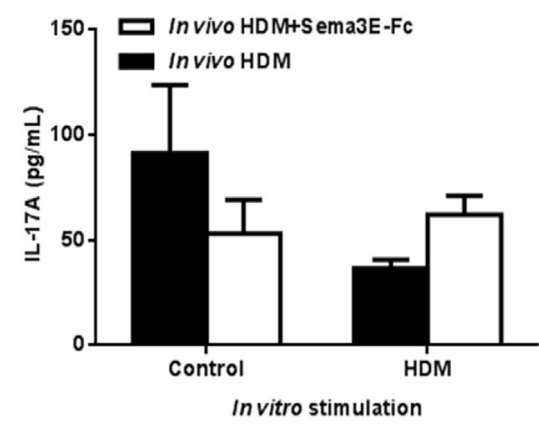

B

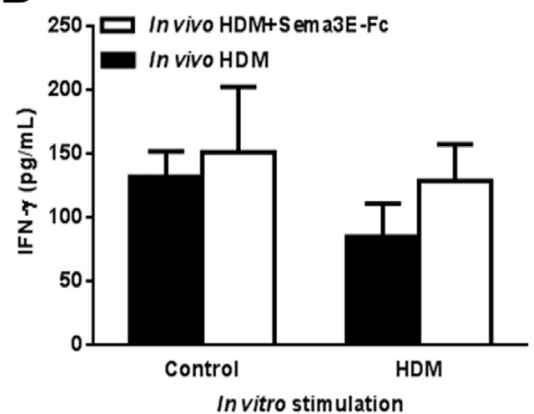

D

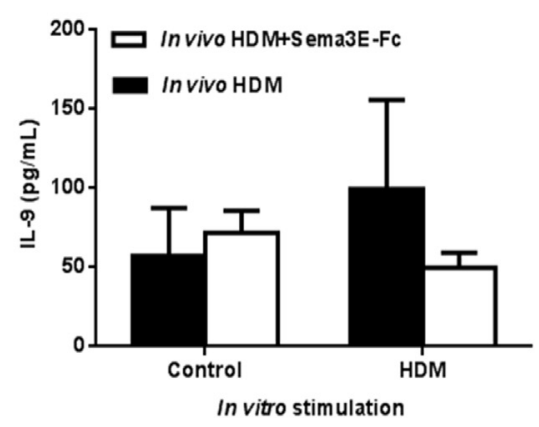

Figure 5 Sema3E treatment induces a sustained regulatory effect on Th1/Th2 balance. Single-cell suspension of mediastinal lymph node (MLN) from the mice exposed to HDM with or without Sema3E-Fc was prepared and stimulated with medium or HDM in vitro. The levels of IL-4 (A), interferon (IFN)- $\gamma$ (B), IL-17A (C), and IL-9 (D) were measured in the supernatant of the cultured cells after 72 hours by enzyme-linked immunosorbent assay. IL-4 secretion induced by in vivo exposure to HDM is inhibited by Sema3E-Fc treatment in MLN cells during in vitro HDM recall. Sema3E treatment does not significantly change secretion of other cytokines in MLN cells. Data are expressed as means \pm SEM. $n=4$ to 6 (A-D). $* * * P<0.001$
The regulatory role of Sema3E in cytokine response encouraged us to examine whether this effect is sustained on HDM recall. MLN single-cell suspensions were isolated from Sema3E-Fc treated and untreated HDM-challenged mice. Then, cells were restimulated with HDM or vehicle in vitro for 72 hours to assess the cytokine response in the supernatant. MLN cells isolated from Sema3E-Fc-treated and HDM-exposed mice had a significant decrease in IL-4 level (Figure 5A) after in vitro HDM recall stimulation compared to that of nontreated mice. But, Sema3E did not significantly alter IFN- $\gamma$ (Figure 5B), IL-17A (Figure 5C), and IL-9 (Figure 5D) levels on HDM restimulation in MLN cells.

To address whether the sustained effect of Sema3E treatment has a potential systemic impact, we studied the cytokine response in splenocytes after HDM recall. Un-stimulated splenocytes from Sema3E-Fc-treated HDMexposed mice had a significantly lower basal level of IL-4 secretion compared to the HDM-exposed group (Supplemental Figure S2A). Furthermore, in vitro stimulation with HDM increased IFN- $\gamma$ level in splenocytes obtained from Sema3E-treated HDM-challenged mice (Supplemental Figure S2B). Contrary to the MLN recall data, Sema3E-Fc reduced IL-17A level in response to HDM restimulation in vitro (Supplemental Figure S2C), whereas the IL-9 level did not significantly change (Supplemental Figure S2D). These results suggest that the Sema3E effect on balancing Th1/Th2 cytokine response is sustained during recall response provoked by HDM.

Next, we measured the level of antibodies involved in regulation of allergic response in the serum obtained from
Sema3E with or without HDM-treated mice. We observed a significant reduction in total (Figure 6A) and HDM-specific (Figure 6B) IgE synthesis on Sema3E-Fc treatment compared to those of Fc-control group. Similarly, both total (Figure 6C) and HDM-specific (Figure 6D) IgG1 levels, elevated by HDM challenge, were diminished after Sema3E treatment. Therefore, the Sema3E suppressive role in allergic airway inflammation could be mediated in part through down-regulation of HDM-induced $\operatorname{IgE}$ and $\mathrm{IgG} 1$ synthesis in the serum.

\section{Sema3E Plays a Crucial Role in Regulation of Pulmonary DC Migration and Function in Allergic Airway Disease}

Pulmonary DC subsets have an essential role in initiation and maintenance of allergic response. To address whether Sema3E implicates in the regulation of pulmonary DC recruitment, we sensitized mice with a single high dose of HDM for 72 hours with or without prior Sema3E treatment. Total $\left(\mathrm{CD} 11 \mathrm{c}^{+} \mathrm{MHCII}^{\mathrm{hi}}\right)$ pulmonary conventional DC (cDC) and also $\mathrm{CD} 103^{+}$subset did not significantly change after Sema3E treatment (Figure 7A). In contrast, HDM-induced $\mathrm{CD} 11 \mathrm{~b}^{+}$pulmonary $\mathrm{cDC}$ recruitment was markedly reduced on Sema3E treatment (Figure 7, A and B).

Because $\mathrm{CD}_{11 b^{+}}$and $\mathrm{CD} 103^{+}$cDCs have been demonstrated to be essential for shaping the immune response to allergens, we purified these cells from HDM with or without Sema3E-Fc treated mice and then investigated their effect on cytokine response on co-culture 

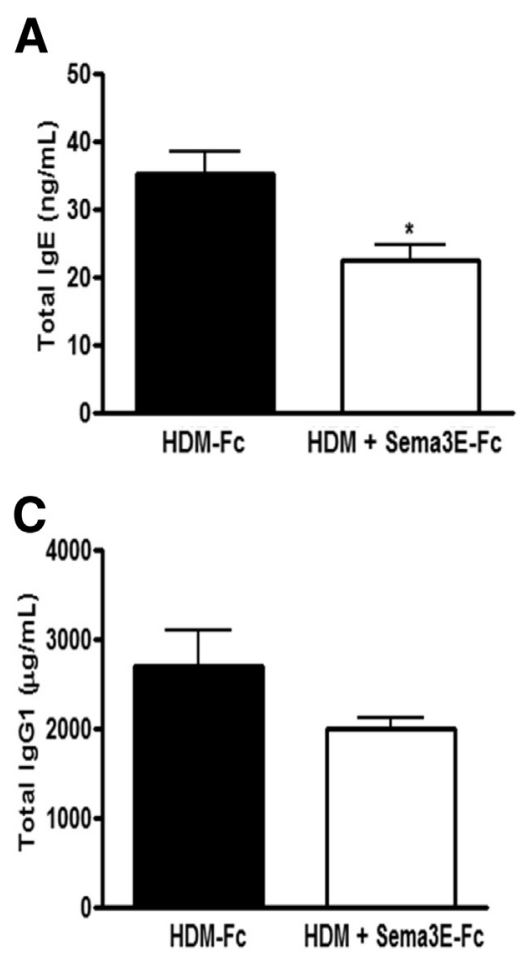

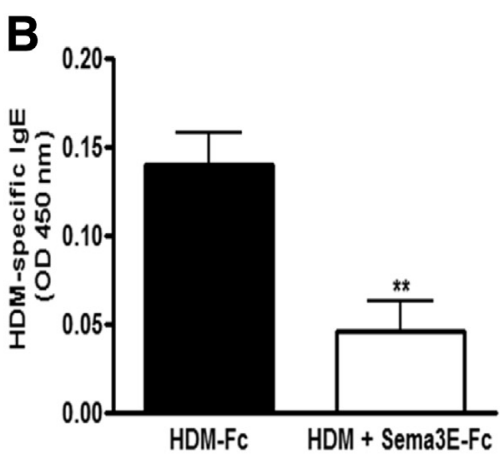

D

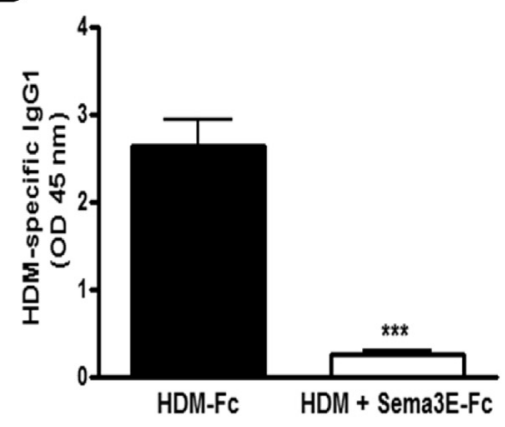

Figure 6 Sema3E treatment regulates allergic antibody response. Sema3E treatment reduces serum levels of both total (A) and HDM-specific (B) IgE as well as IgG1 (C and $\mathbf{D}$ ) provoked by HDM challenge. Data are expressed as means \pm SEM. $n=3$ to 5 (A-D). ${ }^{*} P<0.05,{ }^{*} P<0.01$, and $* * * P<0.001$ versus HDM-Fc. OD, optical density. with $\mathrm{T}$ cells in the presence of $\mathrm{HDM}$ in vitro. $\mathrm{CD} 11 \mathrm{~b}^{+}$ pulmonary cDCs from Sema3E-treated mice induced a significant reduction of IL-5 and IL-17A levels (Figure 7C). On the other hand, despite no effect of Sema3E on the pulmonary recruitment of $\mathrm{CD}_{103}{ }^{+} \mathrm{cDCs}$, it resulted in decreased IL-17A and increased IFN- $\gamma$ levels on HDM stimulation in vitro (Figure 7D). Furthermore, we demonstrated that Sema3E high-affinity receptor is constitutively expressed on the surface of both $\mathrm{CD}_{11} \mathrm{~b}^{+}$and $\mathrm{CD} 103^{+}$ pulmonary cDCs (Figure 7E), suggestive of a functional role on Sema3E binding. Collectively, these data suggest that the effect of Sema3E in allergic asthma could be mediated by regulation of pulmonary $\mathrm{cDC}$ functions.

\section{Discussion}

Semaphorins are ubiquitously expressed and play diverse roles in many biological processes, including immune regulation ${ }^{11,36}$; but our understanding of Sema3E expression and function in allergic asthma has been surprisingly limited. In the present study, we demonstrated that Sema3E expression is reduced in the mouse airways on HDM sensitization and challenge. We further revealed that intranasal administration of recombinant Sema3E protects mice from HDM-induced airway inflammation, remodeling, and AHR. This observation suggests that Sema3E could be considered a promising therapeutic option for allergic airway disease that regulates both inflammatory and structural cell functions.

Sema3E immunoreactivity was clearly observed in the airway epithelium of saline-treated mice, which was considerably reduced on HDM challenge. Down-regulation of Sema3E in allergic asthmatic conditions suggests a crucial role of this mediator in airway homeostasis and allergic inflammation. Similar to Sema3E down-regulation, we previously showed a reduced expression of its high-affinity receptor, PlexinD1, on human ASM cells from allergic asthmatic subjects, ${ }^{28}$ suggesting a dysregulated expression of both ligand and receptor in allergic asthma. Moreover, PlexinD1 was shown to be expressed on $\mathrm{DC}^{23}$ and ASM cells in vitro as well as smooth muscle bundle in bronchial biopsy specimens of allergic asthmatics in vivo. ${ }^{28} \mathrm{We}$ previously revealed that Sema3E modulates growth factor-induced ASM cell proliferation and migration. ${ }^{28}$ Taken together, it is tempting to speculate that Sema3E may modulate airway inflammation by regulating DC function, AHR, and tissue remodeling through inhibiting the migration and proliferation of ASM cells. ${ }^{28}$ This speculation is in accordance with decreased airway inflammation, AHR, and remodeling observed in HDM-challenged and Sema3Etreated mice compared to the vehicle-treated counterpart.

AHR is a major clinical facet of allergic asthma. ${ }^{37}$ Sema3E treatment in mice prevented HDM-induced airway resistance, tissue resistance, and elastance as the characteristic parameters of AHR. A decreased level of IL-4 on Sema3E administration or after recall stimulation may explain, at least in part, the diminished AHR, because IL-4 signaling through STAT6 is required for the development of sustained AHR in mouse models of allergic asthma. ${ }^{38-40}$ Down-regulation of IL-4, as a key player for Ig class switching, by Sema3E treatment may further explain the reduction of proallergic antibody, IgE. ${ }^{41,42}$ 
A
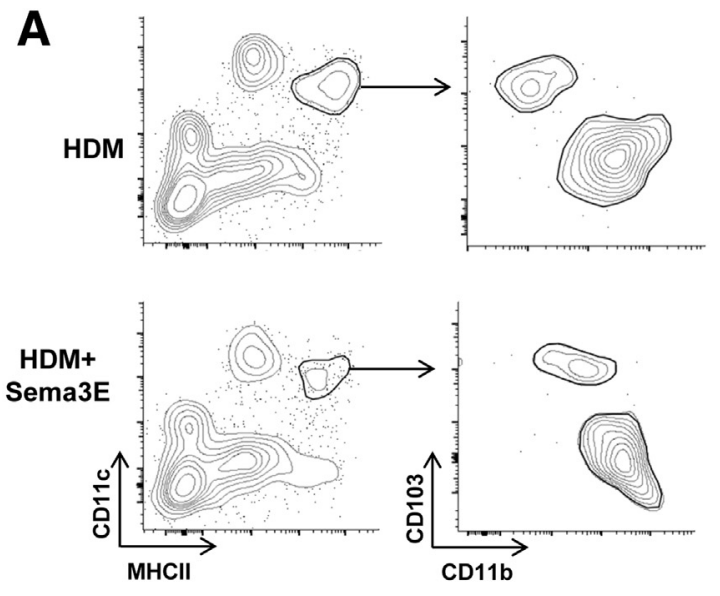

B

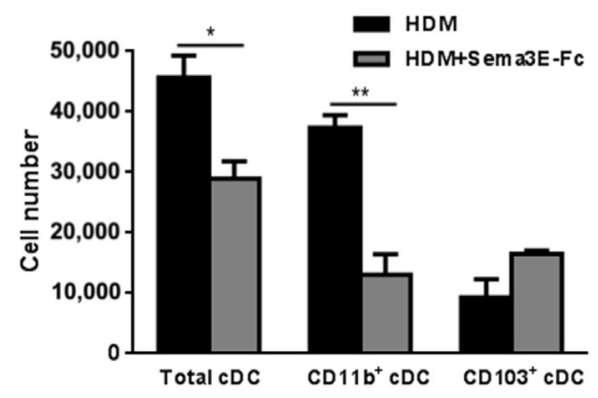

$\mathbf{E}$

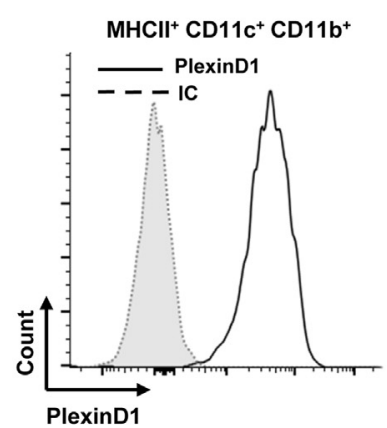

$\mathrm{MHCII}^{+} \mathrm{CD} 11 \mathrm{c}^{+} \mathrm{CD} 103^{+}$

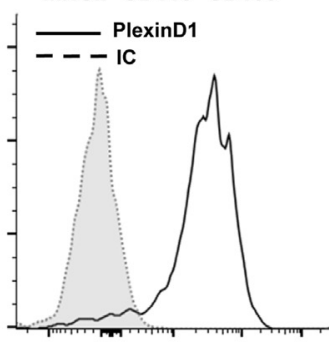

Figure 7 Sema3E treatment regulates pulmonary DC functions. A and B: Intranasal administration of Sema3E decreases the number of total and CD11 $\mathrm{b}^{+}$but not $\mathrm{CD}_{103^{+}}$pulmonary conventional dendritic cells $(\mathrm{CDCS})$, as determined by flow cytometry. $\mathrm{C}$ : $\mathrm{Co}$-culture of Sema3E-treated CD11 $\mathrm{b}^{+}$pulmonary $\mathrm{cDCs}$ obtained from HDM-sensitized mice with T cells reduces IL-5 and IL-17A levels in the supernatant on in vitro HDM stimulation. D: CD103 ${ }^{+}$pulmonary cDCs from the same mice increases interferon (IFN) $-\gamma$, does not change IL-5, and decreases IL-17A levels on co-culture with T cells in the presence of HDM. E: Both CD11 $b^{+}$and $\mathrm{CD}_{103^{+}}$pulmonary CDCs express PlexinD1 on their surface. All data represent two independent experiments. Data are expressed as means $\pm \mathrm{SEM} . n=4(\mathbf{A}-\mathbf{E})$. ${ }^{*} P<0.05,{ }^{*} P<0.01$. IC, isotype control.

Collagen deposition, considered as a key feature of pulmonary fibrosis in chronic asthma, was reduced on Sema3E treatment. There is no report on the role of Sema3E in pulmonary fibrosis, although a recent study reveals that Sema3E secreted by damaged hepatocytes regulates liver fibrosis. ${ }^{21}$ However, Sema7A signaling plays a crucial role in pulmonary fibrosis and remodeling by regulation of phosphatidylinositol 3-kinase/protein kinase B/AKT signaling. ${ }^{43}$ Peng et al ${ }^{44}$ have recently reported that genetic deletion of Sema7A receptor, PlexinC1, leads to increased pulmonary fibrosis. Furthermore, endogenous Sema7A has been shown to inhibit up-regulation of profibrotic genes in human fibroblasts. ${ }^{45}$ Therefore, the effect of Sema3E on pulmonary fibrosis should be further investigated in a chronic model of allergic asthma.

It has been previously revealed that aeroallergens recruit DCs to the airways, and elimination of cDCs has been proposed to prevent allergic airway inflammation and AHR. ${ }^{46,47}$ Herein, we demonstrate that the inhibitory role of Sema3E in experimental allergic asthma could be mediated via modulating recruitment of $\mathrm{CD} 11 \mathrm{~b}^{+} \mathrm{cDCs}$, as Th2 skewing cells, to the airways. Unlike the $\mathrm{CD} 11 \mathrm{~b}^{+} \mathrm{cDC}$ subset, HDM-induced recruitment of $\mathrm{CD} 103^{+}$pulmonary cDCs was not altered by Sema3E. However, our in vitro evidence indicated Sema3Emediated regulation of cytokine response by both $\mathrm{CDC}$ subsets on co-culture with T cells. Sema3E receptor, PlexinD1, has been previously demonstrated to be highly expressed on bone marrow-derived dendritic cells, ${ }^{23}$ as we also showed its surface expression on both pulmonary cDC subsets, which suggests them as responder cells to Sema3E treatment. Decreased recruitment of $\mathrm{CD} 11 \mathrm{~b}^{+} \mathrm{cDCs}$ into the airways of Sema3E-treated allergic mice indicates that Sema3E acts as a guidance cue for these cells, which is analogous to the original function of semaphorins in axon guidance. $^{48}$

In summary, we report that Sema3E plays an essential immunoregulatory role in experimental allergic asthma. Given that Sema3E expression was reduced in allergic asthma and its administration ameliorated the pathological features of the experimental disease, reintroducing Sema3E to the airways might prove efficacious in the treatment of clinical asthma. This previously unknown role for Sema3E in allergic asthma may lead to developing therapeutic strategies in other diseases involving AHR and airway inflammation. 


\section{Acknowledgments}

We thank Dr. Sam Kung and Dr. Thomas Murooka for critical reading of the manuscript, Dr. Christine Zhang (Flow Cytometry Core Facility, University of Manitoba) for her help on sorting lung dendritic cell subsets, Sujata Basu (Murine Lung Function Laboratory, Manitoba Institute of Child Health, Winnipeg, MB, Canada) for her assistance on airway hyperresponsiveness measurements, and Anik Muhuri for his assistance on enzyme-linked immunosorbent assay experiments.

H.M. designed and performed the experiments, analyzed results, and prepared the manuscript; L.S. conducted experiments; J.S.D.-C. cloned, expressed, and purified mouse Semaphorin $3 \mathrm{E}-\mathrm{Fc}$ and revised the manuscript; A.J.H. performed mouse lung mechanics studies; J.E.U. and A.S.G. designed experiments, analyzed data, and prepared the manuscript. All authors revised and approved the final version of the manuscript.

\section{Supplemental Data}

Supplemental material for this article can be found at http://dx.doi.org/10.1016/j.ajpath.2017.03.008.

\section{References}

1. Hekking PP, Bel EH: Developing and emerging clinical asthma phenotypes. J Allergy Clin Immunol Pract 2014, 2:671-680; quiz 681

2. Schatz M, Rosenwasser L: The allergic asthma phenotype. J Allergy Clin Immunol Pract 2014, 2:645-648; quiz 649

3. Barnes PJ: Therapeutic approaches to asthma-chronic obstructive pulmonary disease overlap syndromes. J Allergy Clin Immunol 2015, 136:531-545

4. Darveaux J, Busse WW: Biologics in asthma: the next step toward personalized treatment. J Allergy Clin Immunol Pract 2015, 3: 152-160; quiz 161

5. Fahy JV: Type 2 inflammation in asthma: present in most, absent in many. Nat Rev Immunol 2015, 15:57-65

6. Lauzon AM, Martin JG: Airway hyperresponsiveness: smooth muscle as the principal actor. F1000Res 2016, 5:306

7. Balenga NA, Jester W, Jiang M, Panettieri RA Jr, Druey KM: Loss of regulator of $\mathrm{G}$ protein signaling 5 promotes airway hyperresponsiveness in the absence of allergic inflammation. J Allergy Clin Immunol 2014, 134:451-459

8. Balenga NA, Klichinsky M, Xie Z, Chan EC, Zhao M, Jude J, Laviolette M, Panettieri RA Jr, Druey KM: A fungal protease allergen provokes airway hyper-responsiveness in asthma. Nat Commun 2015, 6:6763

9. McGrath KW, Icitovic N, Boushey HA, Lazarus SC, Sutherland ER, Chinchilli VM, Fahy JV; Asthma Clinical Research Network of the National Heart, Lung, Blood Institute: A large subgroup of mild-tomoderate asthma is persistently noneosinophilic. Am J Respir Crit Care Med 2012, 185:612-619

10. Peters MC, Mekonnen ZK, Yuan S, Bhakta NR, Woodruff PG, Fahy JV: Measures of gene expression in sputum cells can identify TH2-high and TH2-low subtypes of asthma. J Allergy Clin Immunol 2014, 133:388-394

11. Kumanogoh A, Kikutani H: Immunological functions of the neuropilins and plexins as receptors for semaphorins. Nat Rev Immunol 2013, 13:802-814
12. Roth L, Koncina E, Satkauskas S, Cremel G, Aunis D, Bagnard D: The many faces of semaphorins: from development to pathology. Cell Mol Life Sci 2009, 66:649-666

13. Suzuki K, Kumanogoh A, Kikutani H: Semaphorins and their receptors in immune cell interactions. Nat Immunol 2008, 9:17-23

14. He M, Bian Z: Expression of hypoxia-induced semaphorin 7A correlates with the severity of inflammation and osteoclastogenesis in experimentally induced periapical lesions. Arch Oral Biol 2017, 75:114-119

15. Mucka P, Levonyak N, Geretti E, Zwaans BM, Li X, Adini I, Klagsbrun M, Adam RM, Bielenberg DR: Inflammation and lymphedema are exacerbated and prolonged by neuropilin 2 deficiency. Am J Pathol 2016, 186:2803-2812

16. Xie J, Wang H: Semaphorin 7A as a potential immune regulator and promising therapeutic target in rheumatoid arthritis. Arthritis Res Ther 2017, 19:10

17. Xue D, Kaufman GN, Dembele M, Beland M, Massoud AH, Mindt BC, Fiter R, Fixman ED, Martin JG, Friedel RH, Divangahi M, Fritz JH, Mazer BD: Semaphorin 4C protects against allergic inflammation: requirement of regulatory CD138+ plasma cells. J Immunol 2017, 198:71-81

18. Shimizu I, Yoshida Y, Moriya J, Nojima A, Uemura A, Kobayashi Y, Minamino T: Semaphorin3E-induced inflammation contributes to insulin resistance in dietary obesity. Cell Metab 2013, 18:491-504

19. Wanschel A, Seibert T, Hewing B, Ramkhelawon B, Ray TD, van Gils JM, Rayner KJ, Feig JE, O'Brien ER, Fisher EA, Moore KJ: Neuroimmune guidance cue Semaphorin $3 E$ is expressed in atherosclerotic plaques and regulates macrophage retention. Arterioscler Thromb Vasc Biol 2013, 33:886-893

20. Luchino J, Hocine M, Amoureux MC, Gibert B, Bernet A, Royet A, Treilleux I, Lecine P, Borg JP, Mehlen P, Chauvet S, Mann F: Semaphorin $3 \mathrm{E}$ suppresses tumor cell death triggered by the plexin D1 dependence receptor in metastatic breast cancers. Cancer Cell 2013, 24:673-685

21. Yagai T, Miyajima A, Tanaka M: Semaphorin 3E secreted by damaged hepatocytes regulates the sinusoidal regeneration and liver fibrosis during liver regeneration. Am J Pathol 2014, 184:2250-2259

22. Hughes A, Kleine-Albers J, Helfrich MH, Ralston SH, Rogers MJ: A class III semaphorin (Sema3e) inhibits mouse osteoblast migration and decreases osteoclast formation in vitro. Calcif Tissue Int 2012, 90: $151-162$

23. Holl EK, Roney KE, Allen IC, Steinbach E, Arthur JC, Buntzman A, Plevy S, Frelinger J, Ting JP: Plexin-B2 and Plexin-D1 in dendritic cells: expression and IL-12/IL-23p40 production. PLoS One 2012, 7: e43333

24. Choi YI, Duke-Cohan JS, Ahmed WB, Handley MA, Mann F, Epstein JA, Clayton LK, Reinherz EL: PlexinD1 glycoprotein controls migration of positively selected thymocytes into the medulla. Immunity 2008, 29:888-898

25. Moriya J, Minamino T, Tateno K, Okada S, Uemura A, Shimizu I, Yokoyama M, Nojima A, Okada M, Koga H, Komuro I: Inhibition of semaphorin as a novel strategy for therapeutic angiogenesis. Circ Res 2010, 106:391-398

26. Sabag AD, Bode J, Fink D, Kigel B, Kugler W, Neufeld G: Semaphorin-3D and semaphorin-3E inhibit the development of tumors from glioblastoma cells implanted in the cortex of the brain. PLoS One 2012, 7:e42912

27. Sakurai A, Gavard J, Annas-Linhares Y, Basile JR, Amornphimoltham P, Palmby TR, Yagi H, Zhang F, Randazzo PA, Li X, Weigert R, Gutkind JS: Semaphorin 3E initiates antiangiogenic signaling through plexin D1 by regulating Arf6 and R-Ras. Mol Cell Biol 2010, 30:3086-3098

28. Movassagh H, Shan L, Halayko AJ, Roth M, Tamm M, Chakir J, Gounni AS: Neuronal chemorepellent Semaphorin 3E inhibits human airway smooth muscle cell proliferation and migration. J Allergy Clin Immunol 2014, 133:560-567

29. Movassagh H, Shan L, Mohammed A, Halayko AJ, Gounni AS: Semaphorin 3E deficiency exacerbates airway inflammation, 
hyperresponsiveness, and remodeling in a mouse model of allergic asthma. J Immunol 2017, 198:1805-1814

30. Movassagh H, Saati A, Nandagopal S, Mohammed A, Tatari N, Shan L, Duke-Cohan JS, Fowke KR, Lin F, Gounni AS: Chemorepellent semaphorin $3 \mathrm{E}$ negatively regulates neutrophil migration in vitro and in vivo. J Immunol 2017, 198:1023-1033

31. Hirota JA, Budelsky A, Smith D, Lipsky B, Ellis R, Xiang YY, Lu WY, Inman MD: The role of interleukin-4Ralpha in the induction of glutamic acid decarboxylase in airway epithelium following acute house dust mite exposure. Clin Exp Allergy 2010, 40:820-830

32. Gounni AS, Spanel-Borowski K, Palacios M, Heusser C, Moncada S, Lobos E: Pulmonary inflammation induced by a recombinant Brugia malayi gamma-glutamyl transpeptidase homolog: involvement of humoral autoimmune responses. Mol Med 2001, 7:344-354

33. Plantinga M, Guilliams M, Vanheerswynghels M, Deswarte $K$, Branco-Madeira F, Toussaint W, Vanhoutte L, Neyt K, Killeen N, Malissen B, Hammad H, Lambrecht BN: Conventional and monocytederived $\mathrm{CD} 1 \mathrm{~b}(+)$ dendritic cells initiate and maintain $\mathrm{T}$ helper 2 cellmediated immunity to house dust mite allergen. Immunity 2013, 38: 322-335

34. Fattouh $\mathrm{R}$, Al-Garawi A, Fattouh $\mathrm{M}$, Arias $\mathrm{K}$, Walker TD, Goncharova S, Coyle AJ, Humbles AA, Jordana M: Eosinophils are dispensable for allergic remodeling and immunity in a model of house dust mite-induced airway disease. Am J Respir Crit Care Med 2011, $183: 179-188$

35. Johnson JR, Wiley RE, Fattouh R, Swirski FK, Gajewska BU, Coyle AJ, Gutierrez-Ramos JC, Ellis R, Inman MD, Jordana M: Continuous exposure to house dust mite elicits chronic airway inflammation and structural remodeling. Am J Respir Crit Care Med 2004, 169:378-385

36. Worzfeld T, Offermanns S: Semaphorins and plexins as therapeutic targets. Nat Rev Drug Discov 2014, 13:603-621

37. Busse WW: The relationship of airway hyperresponsiveness and airway inflammation: airway hyperresponsiveness in asthma: its measurement and clinical significance. Chest 2010, 138:4S-10S

38. Chapoval SP, Dasgupta P, Smith EP, DeTolla LJ, Lipsky MM, KellyWelch AE, Keegan AD: STAT6 expression in multiple cell types mediates the cooperative development of allergic airway disease. J Immunol 2011, 186:2571-2583

39. Leigh R, Ellis R, Wattie JN, Hirota JA, Matthaei KI, Foster PS, O'Byrne PM, Inman MD: Type 2 cytokines in the pathogenesis of sustained airway dysfunction and airway remodeling in mice. Am J Respir Crit Care Med 2004, 169:860-867

40. Sahoo A, Alekseev A, Obertas L, Nurieva R: Grail controls Th2 cell development by targeting STAT6 for degradation. Nat Commun 2014, $5: 4732$

41. Erazo A, Kutchukhidze N, Leung M, Christ AP, Urban JF Jr, Curotto de Lafaille MA, Lafaille JJ: Unique maturation program of the $\operatorname{IgE}$ response in vivo. Immunity 2007, 26:191-203

42. Wu LC, Zarrin AA: The production and regulation of IgE by the immune system. Nat Rev Immunol 2014, 14:247-259

43. Kang HR, Lee CG, Homer RJ, Elias JA: Semaphorin 7A plays a critical role in TGF-betal-induced pulmonary fibrosis. J Exp Med 2007, 204:1083-1093

44. Peng X, Moore M, Mathur A, Zhou Y, Sun H, Gan Y, HerazoMaya JD, Kaminski N, Hu X, Pan H, Ryu C, Osafo-Addo A, Homer RJ, Feghali-Bostwick C, Fares WH, Gulati M, Hu B, Lee CG, Elias JA, Herzog EL: Plexin C1 deficiency permits synaptotagmin 7mediated macrophage migration and enhances mammalian lung fibrosis. FASEB J 2016, 30:4056-4070

45. Esnault S, Torr EE, Bernau K, Johansson MW, Kelly EA, Sandbo N, Jarjour NN: Endogenous semaphorin-7A impedes human lung fibroblast differentiation. PLoS One 2017, 12:e170207

46. Bratke K, Lommatzsch M, Julius P, Kuepper M, Kleine HD, Luttmann W, Christian Virchow J: Dendritic cell subsets in human bronchoalveolar lavage fluid after segmental allergen challenge. Tho$\operatorname{rax} 2007,62: 168-175$

47. Moran TP, Nakano K, Whitehead GS, Thomas SY, Cook DN, Nakano H: Inhaled house dust programs pulmonary dendritic cells to promote type 2 T-cell responses by an indirect mechanism. Am J Physiol Lung Cell Mol Physiol 2015, 309:L1208-L1218

48. Yoshida Y: Semaphorin signaling in vertebrate neural circuit assembly. Front Mol Neurosci 2012, 5:71 